\title{
Article \\ The Effect of Rainfall on the UAV Placement for 5G Spectrum in Malaysia
}

\author{
Abdulrahman M. Shalaby (D) and Noor Shamsiah Othman *(D) \\ Department of Electrical and Electronics Engineering, Universiti Tenaga Nasional, \\ Kajang 43000, Selangor, Malaysia; Abdulrahman@uniten.edu.my \\ * Correspondence: Shamsiah@uniten.edu.my
}

check for updates

Citation: Shalaby, A.M.; Othman,

N.S. The Effect of Rainfall on the UAV Placement for 5G Spectrum in Malaysia. Electronics 2022, 11, 681. https://doi.org/10.3390/

electronics11050681

Academic Editor: Nurul I. Sarkar

Received: 24 October 2021

Accepted: 22 November 2021

Published: 23 February 2022

Publisher's Note: MDPI stays neutral with regard to jurisdictional claims in published maps and institutional affiliations.

Copyright: (c) 2022 by the authors. Licensee MDPI, Basel, Switzerland. This article is an open access article distributed under the terms and conditions of the Creative Commons Attribution (CC BY) license (https:// creativecommons.org/licenses/by/ $4.0 /)$.

\begin{abstract}
In this paper, the influence of rainfall on the deployment of UAV as an aerial base station in the Malaysia 5G network is studied. The outdoor-to-outdoor and outdoor-to-indoor path loss models are derived by considering the user's antenna height, rain attenuation, and the wall penetration loss at high frequencies. The problem of finding the UAV 3D placement is formulated with the objective to minimize the total path loss between the UAV and all users. The problem is solved by invoking two algorithms, namely Particle Swarm Optimization (PSO) and Gradient Descent (GD) algorithms. The performance of the proposed algorithms is evaluated by considering two scenarios to determine the optimum location of the UAV, namely outdoor-to-outdoor and outdoor-to-indoor scenarios. The simulation results show that, for the outdoor-to-outdoor scenario, both algorithms resulted in similar UAV 3D placement unlike for the outdoor-to-indoor scenario. Additionally, in both scenarios, the proposed algorithm that invokes PSO requires less iterations to converge to the minimum transmit power compared to that of the algorithm that invokes GD. Moreover, it is also observed that the rain attenuation increases the total path loss for high operating frequencies, namely at $24.9 \mathrm{GHz}$ and 28.1 GHz. Hence, this resulted in an increase of UAV required transmit power. At $28.1 \mathrm{GHz}$, the presence of rain at the rate of $250 \mathrm{~mm} / \mathrm{h}$ resulted in an increase of UAV required transmit power by a factor of 4 and 15 for outdoor-to-outdoor and outdoor-to-indoor scenarios, respectively.
\end{abstract}

Keywords: 5G; wireless communication; particle swarm optimization; gradient descent; path loss; line of sight probability; rain attenuation; unmanned aerial vehicles (UAV); aerial base station

\section{Introduction}

There are many reviews on the challenges of communication system during disasters. A review on the readily available communication system in Malaysia and its vulnerability during disaster was presented in [1]. It was highlighted that an efficient emergency communication system during disaster is required to speed up relief and recovery operations. Moreover, in the event of natural disaster, communication through mobile networks outages may lead to network congestion. One of the solutions is to deploy UAV as an aerial base station $[2,3]$.

The deployment of UAV as an aerial based station can be due to the cellular outage, due to disaster situation [4] or as a supplement to the ground base station to provide better coverage during an event [5]. The effective deployment of UAV requires meeting some of the key challenges such as air-to-ground channel modeling, optimal deployment, path planning, and energy-efficient operation [6]. More specifically, in this situation, the effective deployment of UAV is derived such that it provides coverage for indoor users with minimum transmit power derived by employing different heuristic algorithms [7].

There has been an increase of research interest in the efficient 3D deployment of UAV strategies to provide wireless coverage to outdoor ground users only, indoor ground users only or outdoor and indoor ground users simultaneously. This is because the UAV placement problem has an impact on the power consumption $[5,8,9]$. Thus, this 
motivates research interest in the efficient 3D deployment of UAVs strategies that aim for minimum transmit power. The efficient 3D deployment of UAVs addresses some of the key technical challenges such as power consumption, wireless coverage optimization, interference management, and users' experienced data rate [8,10-13].

In $[2,3]$, the authors presented the classification of UAV deployment strategies based on objective functions, namely, minimizing the transmit power of UAVs [6,14], maximizing the wireless coverage of UAVs [10], and minimizing the number of UAVs required to perform a given task [15], and optimizing UAV trajectory [16]. However, in this study, the optimal UAV 3D placement is formulated with the objective to minimize the total path loss between the UAV and the users.

Due to the shortage in frequency spectrum allocated to a $4 \mathrm{G}$ wireless communication system operating at lower frequencies than $6 \mathrm{GHz}$, several frequency bands have been added and the frequency spectrum has been extended to millimeter waves (mmWaves) so as to support a $5 \mathrm{G}$ communication system with a countless number of services that need a lot of bandwidth to deliver a high data rate to the end-user. mmWaves are suffering from higher attenuation, compared to lower bands, because of energy absorption and scattering by meteorological conditions such as rainfall $[17,18]$.

Rain attenuation is one of the major obstacles in propagation of mmWaves in $5 \mathrm{G}$ systems [19]. It can be a significant factor depending upon the link distance and the geographic location as in Malaysia is considered as a critical location due its location in tropical regions that experience heavy rains with large raindrop sizes [20,21]. Thus, the effect of rain attenuation to 5G spectrum in Malaysia must be studied. Based on ITU-R P.838-3 model [22], the rain attenuation becomes critical with the horizontal polarization type (worst case), which resulted in more attenuation compared to vertical polarization.

In [17], the authors used three years of raindrop size distribution (DSD) data collected in Kuala Lumpur, Malaysia to calculate rain attenuation and compared to the one available in ITU-R P.838-3. The performance of the model can be used to predict the rain attenuation of $5 \mathrm{G}$ wireless communication systems in heavy rain regions. However, a majority of the studies on the effect of rainfall on electromagnetic wave propagation were performed experimentally $[17,20,21]$.

Path loss models are essential elements for the design and analysis of UAV communication channels [23]. The path loss model describes the amount of reduction in power density of a transmitted signal as it propagates through the channel. Several outdoor-tooutdoor path loss models in a dense urban area have been studied in [24-26]. The path loss model in [24] is derived by performing simulations for three different frequencies, namely, $700 \mathrm{MHz}, 2000 \mathrm{MHz}$, and $5800 \mathrm{MHz}$. In this study, the influence of rainfall is studied when considering UAV deployment as an aerial base station to provide wireless coverage for two scenarios where users are located outdoor only and users are located outdoors and indoors for a 5G spectrum. Thus, the path loss model must be modified to consider higher operating frequencies and rain attenuation. Similar modification must also be done for the outdoor-to-indoor path loss scenario.

However, the deployment of UAV as an aerial base station in the previous studies $[6,8,15,16]$ did not consider the effect of rain attenuation for urban environment on the propagation of mmWaves. Therefore, in this paper, the influence of rainfall on the deployment of UAV as aerial base station in Malaysia 5G network is studied. Thus, the outdoor-to-outdoor and outdoor-to-indoor path loss models that include rain attenuation are derived. Moreover, in this paper, the probability of Line-of-sight (LoS) that considers a user's antenna height and the wall penetration loss operating at high frequency bands are derived and included in the path loss models. More specifically, the derived path loss models are used in the proposed algorithms to find the UAV 3D placement by invoking particle swarm optimization (PSO) and gradient descent (GD) algorithms.

The contributions of this work can be summarized as follows:

1. Line-of-sight $(L o S)$ probability that is applicable for users at a higher level above the ground and suitable for different environments which depends on parameters related 
to the area served by UAV coverage is derived. As the height of the user's antenna increases, the probability of $L o S$ increases and hence there is a reduction in path loss.

2. Derivation of wall penetration loss that considers operating frequency at higher bands for the case when a signal traverses a wall that consists of two materials, namely concrete and glass.

3. The rain attenuation and its effect in path loss model for both outdoor and indoor users at mmWaves frequencies is contrived.

4. The optimal UAV 3D placement algorithms that invoke GD and PSO algorithms are developed with the objective to minimize the total path loss between the UAV and all users for two scenarios, namely outdoor-to-outdoor and outdoor-to-indoor. The newly derived path loss models that consider the user's antenna heights, wall penetration loss, and rain attenuation are utilized to study the influence of rainfall on the deployment of UAV.

The rest of this paper is organized as follows: Section 2 describes the system models which includes the derivation of path loss models for outdoor and indoor users that considers user's antenna height, rain attenuation, and wall penetration loss for operating frequencies at higher bands. The derivation of the probability of $L o S$, rain attenuation, and wall penetration loss at frequencies in $5 \mathrm{G}$ spectrum are also presented in this section. The main components of path loss for both outdoor and indoor users.

Section 2.3 focuses on the development of UAV 3D placement algorithms that invoke the GD and PSO algorithms, while the performance of the proposed algorithms is evaluated for two scenarios, and the simulation results and discussion are presented in Section 3. Finally, the conclusions are presented in Section 4.

\section{System Model}

This study considers the deployment of a single UAV that provides wireless coverage to outdoor and indoor users within a square coverage area of $150 \mathrm{~m} \times 150 \mathrm{~m}$. The coverage area consists of four buildings with a park located at the center of the area, as shown in Figure 1. The 3D placement of the UAV is denoted as $\left(x_{U A V}, y_{U A V}, z_{U A V}\right)$ and $\left(x_{i}, y_{i}\right)$ denotes the location of the user $i$.

In this study, the deployment of UAV as aerial base station considers its deployment at a low altitude platform (LAP) $[27,28]$ for two scenarios, namely, outdoor-to-outdoor and outdoor-to-indoor scenarios. In the outdoor-to-outdoor scenario, all users are located outdoors, whilst, in the outdoor-to-indoor scenario, users are located outdoors and indoors.

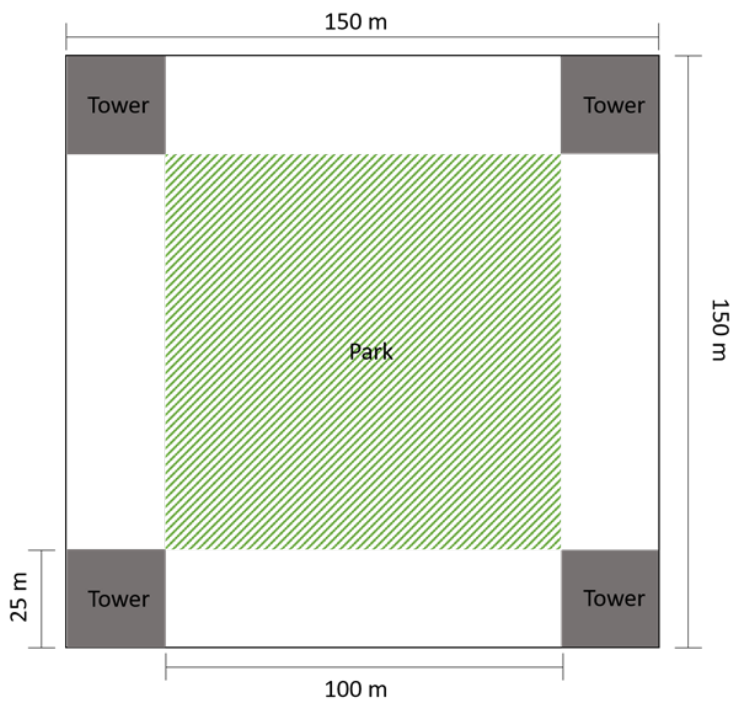

Figure 1. UAV wireless coverage area. 


\subsection{Outdoor-to-Outdoor Path Loss Model}

The outdoor-to-outdoor scenario considers the deployment of UAV as an aerial base station in providing wireless coverage to outdoor users only. The air-to-ground (ATG) path loss model in [24] predicts the path loss between LAP and a ground receiver. It is modeled by considering the probabilistic mean path loss, which is averaged over the Line-of-Sight (LoS) and Non-Line-of-Sight (NLoS) conditions.

However, the probability of LoS in the path loss model of [24] does not consider user's antenna height. It was observed that the probability of LoS increased, as the user's antenna height increased [29]. Therefore, in this work, the LoS probability that takes into consideration the user's antenna height is derived and included in the outdoor-to-outdoor path loss model.

Moreover, in this study, the effect of rain attenuation to 5G spectrum in Malaysia is studied. Therefore, the rain attenuation, $L_{p}$ is derived and included in the outdoor-tooutdoor path loss model as follows:

$$
L_{O}=20 \log \left(\frac{4 \pi f_{c}}{c}\right)+20 \log \left(\sqrt{h^{2}+r^{2}}\right)+P(\operatorname{LoS}) \eta_{L o S}+P(N L o S) \eta_{N L o S}+L_{p}
$$

where $f_{c}$ is the carrier frequency, $c$ is the speed of the light, $h$ is the UAV height, $r$ is the horizontal distance between the UAV and the user, $\eta_{L O S}$ and $\eta_{N L O S}$ are the average additional loss which depends on the environment, and $P(L o S)$ and $L_{p}$ are given by Equations (6) and (11), respectively.

\subsubsection{Line of Sight Probability}

Figure 2 shows the system model to calculate the $P(L o S)$ considering the user's antenna height, where the aerial base station antenna height is denoted by $h_{U A V}$, the user's antenna height is referred to as $h_{u}, d$ is the horizontal distance from the aerial base station, $W_{s}$ is the average street width and $h_{b}$ is the building height. Using triangles similarity, the relationship between the building height, $h_{b o}$ and the aerial base station antenna height, $h_{U A V}$ at the onset of $L O S$ can be written as:

$$
\frac{h_{u}}{h_{U A V}}=\frac{x}{x+d}
$$

and

$$
\frac{x}{x+\left(W_{S} / 2\right)}=\frac{h_{u}}{h_{b o}}
$$

where $h_{b o}$ is the building height at the onset of LoS. Thus, $h_{b o}$ can be re-written as Equation (4) by solving these two above equations, and eliminating $x$ :

$$
h_{b o}=\left(\left(W_{s} / 2 h_{U A V}+h_{u}\right)\left(d-\left(W_{s} / 2\right)\right)\right) / d
$$

One of the most important conditions in an urban environment is the layout and characteristics of the building. The parameter that describes the geometrical statistics of a certain urban area of which the RF signal propagates is $\gamma$ [29]. This parameter describes the buildings heights distribution according to Rayleigh distribution function [29]:

$$
\left.P\left(h_{b}\right)=\left(h_{b} e^{\left(-\left(h_{b}^{2}\right) /\left(2 \gamma^{2}\right)\right.}\right) / \gamma^{2}\right)
$$

where $h_{b}$ is the building height in meters.

Equation (5) is used to plot Figure 3. It is clear that increasing the value of $\gamma$ results in a higher range of building heights distribution. For $\gamma=6$, the building height at the onset of $L o S$ can be calculated using Equation (4), which gives the value of $P\left(h_{b}\right)=0.06$, which is illustrated as the vertical line in Figure 3. 


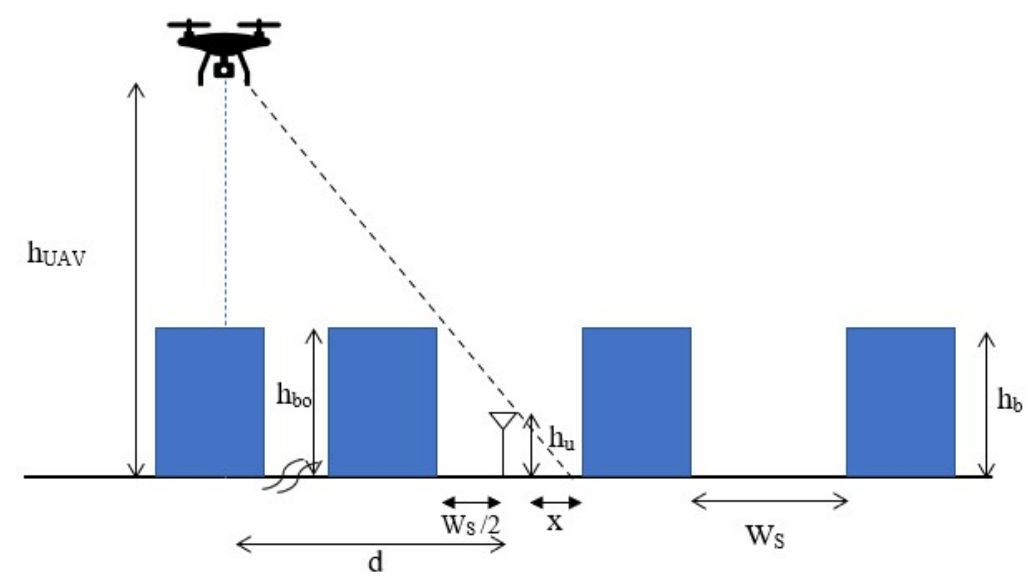

Figure 2. System model to calculate the $P(L o S)$ considering the user's antenna height.

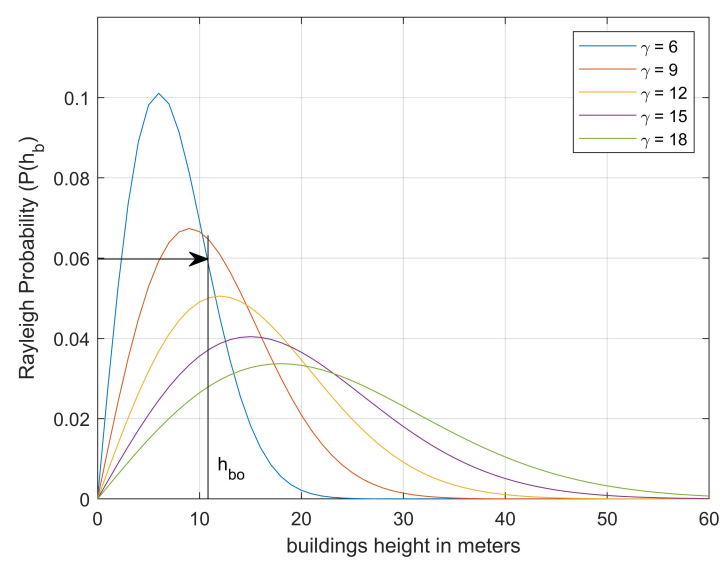

Figure 3. The Rayleigh probability density function distribution versus building heights for different values of the parameter $\gamma$.

Thus, the probability of $L o S$ equals the area under the curve from $h_{b}=0$ to $h_{b}=h_{b o}$, which is given as

$$
P(L o S)=\int_{0}^{h_{b o}} P\left(h_{b}\right) d h_{b}=\int_{0}^{h_{b o}}\left(h_{b} e^{-\left(h_{b}^{2}\right) /\left(2 \gamma^{2}\right)}\right) / \gamma^{2} d h_{b}=\left(1-e^{-\left(h_{b o}^{2}\right) /\left(2 \gamma^{2}\right)}\right)
$$

The effects of user's antenna and UAV height on the probability of LoS can be analyzed using Equations (4) and (6).

Line of Sight Probability Analysis and Validation

Figure 4a shows the LoS probability versus the UAV height for different values of the horizontal distance between the UAV and the user that are located within the UAV coverage area when the user antenna height is at the ground level, $\gamma=6$ and street average width of $20 \mathrm{~m}$, which is an estimation for urban environment. This figure shows that the probability of LoS increases as the UAV height increases for the same horizontal distance between the UAV and the user. On the other hand, the probability of LoS decreases as the horizontal distance from the UAV increases for the same UAV height.

Figure $4 \mathrm{~b}$ shows the effect of increasing the user's antenna height on the probability of LoS keeping the height of the UAV constant at $50 \mathrm{~m}$ for the same parameters as Figure $4 \mathrm{a}$ where $\gamma=6$ and street average width is $20 \mathrm{~m}$. As the height of the user's antenna increases, the probability of $L o S$ increases and hence decreases the path loss, thus contributing as a gain factor as indicated in [29]. The increase in LoS probability and hence reduction in path loss is more significant for lower values of horizontal distances between the UAV and the user. 


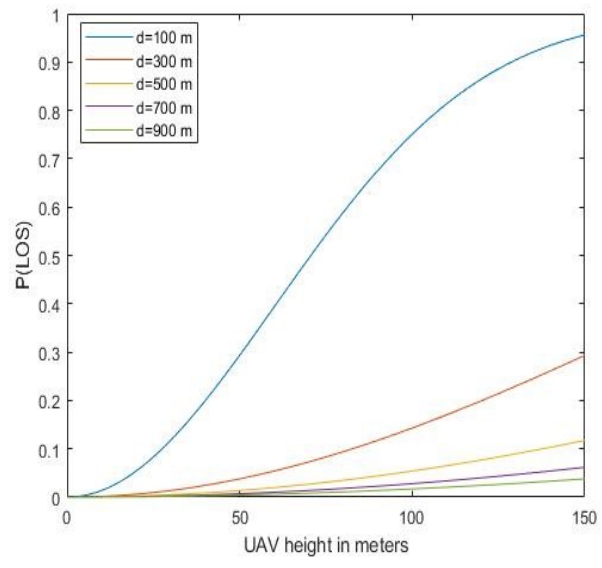

(a)

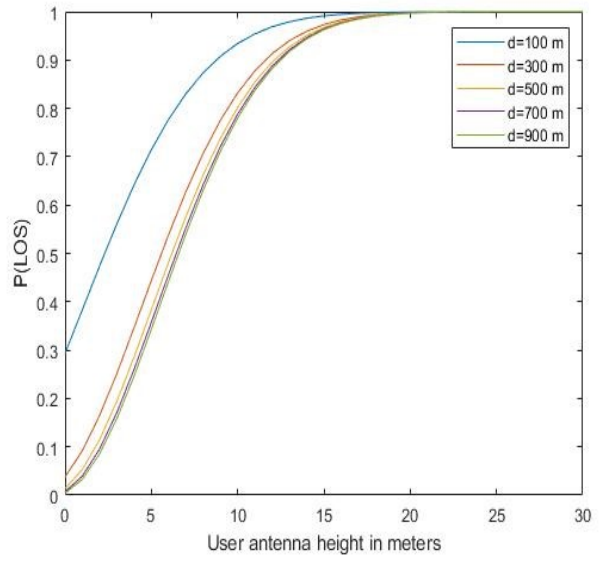

(b)

Figure 4. The probability of LoS for different horizontal distances, $d$ between the user and the UAV. (a) $\mathrm{P}(L o S)$ versus UAV height when the user's antenna is at ground level; (b) $\mathrm{P}(L o S)$ versus user's antenna height when the UAV height $=50 \mathrm{~m}$.

The $\mathrm{P}(L o S)$ Equation (6) is validated with the work by the authors in [24]. The value of parameters in [24] are substituted into their $\mathrm{P}(L o S)$ model to plot Figure 5a-c. The dotted line in these figures shows the plot using Al-Hourani's $\mathrm{P}(\operatorname{LoS})$ model of [24], whereas the solid line shows the $\mathrm{P}(L o S)$ plot of Equation (6). The value of variables $\gamma$ and $W_{S}$ varies with different environments dense urban, urban, and suburban as shown in Table 1.

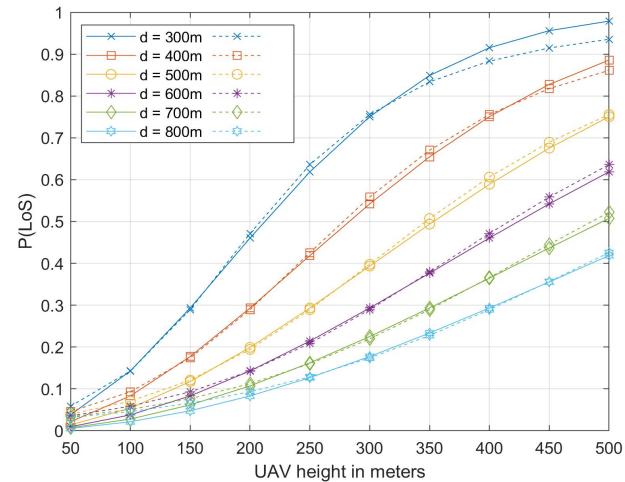

(a)

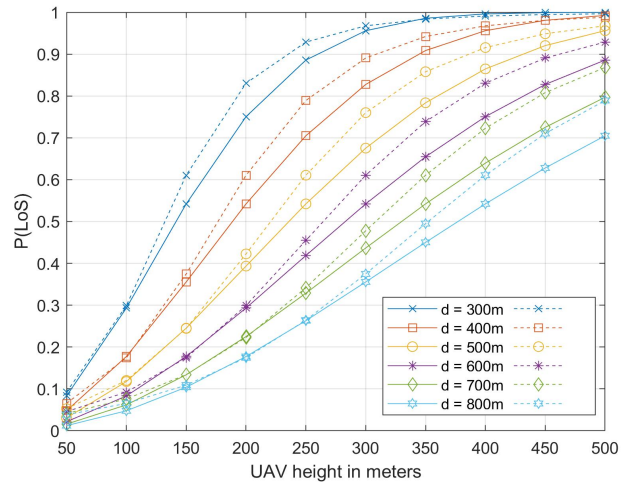

(b)

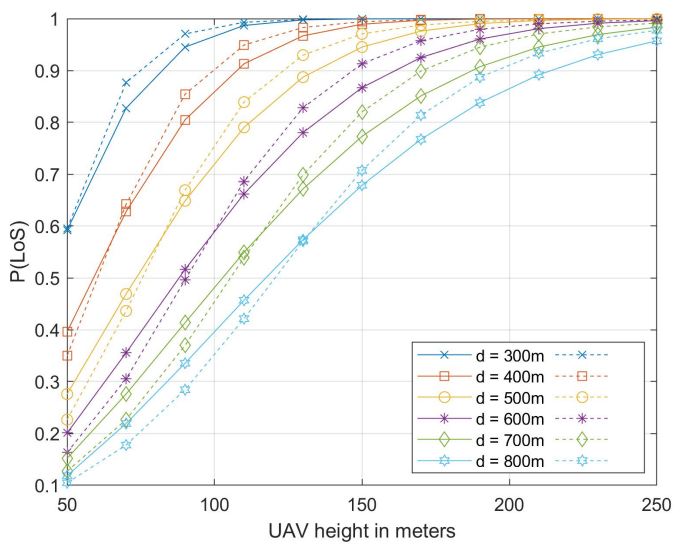

(c)

Figure 5. Probability LoS versus UAV height in meters for different $d$ (a) in a dense urban environment; (b) in an urban environment; (c) in a suburban environment. 
Table 1. Summary of value of gamma, $\gamma$ and $W_{s}$ for different environments.

\begin{tabular}{ccc}
\hline & $\boldsymbol{W}_{\boldsymbol{s}}$ & $\boldsymbol{\gamma}$ \\
\hline Suburban & $45 \mathrm{~m}$ & 2.8 \\
Urban & $30 \mathrm{~m}$ & 6 \\
Dense urban & $20 \mathrm{~m}$ & 6 \\
\hline
\end{tabular}

It can be seen from Figure 5a-c that the $P(L o S)$ of Equation (6) is in agreement with that of Al-Hourani's for dense urban environment. For the case of urban environment, the $P(L o S)$ of Equation (6) is in agreement with that of Al-Hourani's. Meanwhile, for the case of a suburban environment, the $P(L o S)$ of Equation (6) is in close agreement with that of Al-Hourani's.

\subsubsection{Rain Attenuation}

Based on ITU rain regions for Asia in [30], Malaysia falls in the P region and the rainfall intensity exceeded in $\mathrm{mm} / \mathrm{h}$ as a percentage of time of the year (with a 1-min integration time) for $\mathrm{P}$ region is shown in Table 2.

Table 2. Rainfall intensity exceeded $(\mathrm{mm} / \mathrm{h})$ for the climate zone of Malaysia [30].

\begin{tabular}{cc}
\hline $\begin{array}{c}\text { Percentage of Time } \\
(\%)\end{array}$ & $\begin{array}{c}\text { Rainfall Intensity Exceeded } \\
(\mathbf{m m} / \mathbf{h})\end{array}$ \\
\hline 1.0 & 12 \\
0.3 & 34 \\
0.1 & 65 \\
0.03 & 105 \\
0.01 & 145 \\
0.003 & 200 \\
0.001 & 250 \\
\hline
\end{tabular}

Specific rain attenuation based on rain rates can be calculated following the procedure recommended by the ITU-R [22,30]. The specific attenuation $\gamma_{R}(\mathrm{~dB} / \mathrm{km})$ is obtained from the rain rate $R(\mathrm{~mm} / \mathrm{h})$ using the power-law relationship [22,30]:

$$
\gamma_{R}=k R^{\alpha}
$$

where the value of the coefficients $k$ and $\alpha$ are given as function of frequency, $f(\mathrm{GHz})$, in the range from 1 to $1000 \mathrm{GHz}$, for different polarization tilt angle relative to the horizontal, and for a different path elevation angle from [22].

For a rain rate exceeding $0.01 \%$ of the time, the attenuation due to rain $L_{R}$ in $\mathrm{dB}$ can be calculated using the formula:

$$
L_{R 0.01}=k R^{\alpha} d r
$$

where $d$ is the link distance in $\mathrm{km}$ and the coefficient $r$ is calculated as follows:

$$
r=\frac{1}{1+\frac{d}{d_{0}}}
$$

and the coefficient $d_{0}$ in $\mathrm{km}$ for $R_{0.01} \leq 100 \mathrm{~mm} / \mathrm{h}$ is given by:

$$
d_{0}=35 e^{-0.015 R_{0.01}}
$$

where for $R_{0.01}>100 \mathrm{~mm} / \mathrm{h}$, the $R_{0.01}$ value of $100 \mathrm{~mm} / \mathrm{h}$ is substituted in Equation (10). For percentages of time $p$ other than 0.01, the attenuation is calculated using Equation (11), which is suitable for Malaysia:

$$
L_{p}=L_{R 0.01} 0.12 p^{-\left(0.546+0.043 \log _{10} p\right),}
$$


where $p$ ranges from 0.001 to $1 \%$.

The specific rain attenuation analysis is performed using Equation (11). Figure 6 shows the dependence of the rain attenuation on the rain rate for the frequencies of interest of the mobile network in Malaysia. It also shows that increasing the carrier frequency leads to an increase in the attenuation value, whereas Figure 7 shows the dependence of the rain attenuation on the frequencies of interest, for the different rain rates registered in Malaysia for one year that ranges from 0 to $250 \mathrm{~mm} / \mathrm{h}$ [30].

At low frequencies, a different value of rain rate does not significantly cause rain attenuation, but it will be noticeable at higher frequencies ( $\mathrm{mm}$ Waves). The propagation loss through rain reaches a value of $40 \mathrm{~dB} / \mathrm{km}$ at $24.9 \mathrm{GHz}$ operating frequency which is high and should be taken into consideration in any design problem of UAV deployment at 5G frequencies in Malaysia.

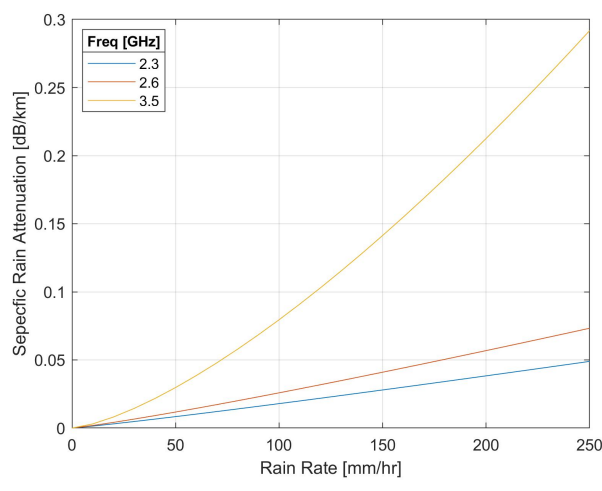

(a)

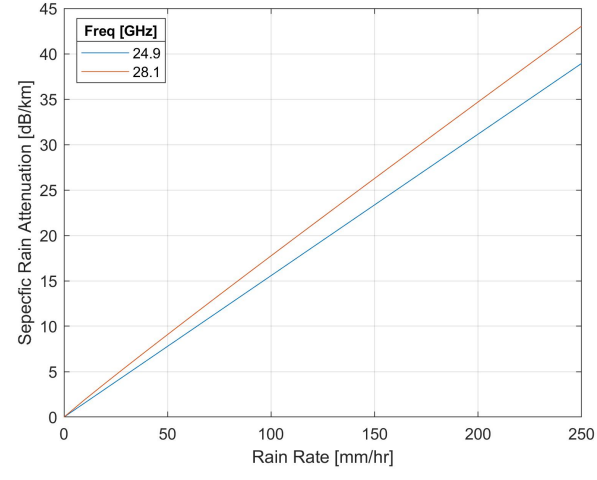

(b)

Figure 6. Rain attenuation versus rain rate values registered during one year in Malaysia. (a) for lower frequencies 2.3, 2.6, and 3.5 GHz; (b) for higher frequencies, 24.9 and 28.1 GHz.

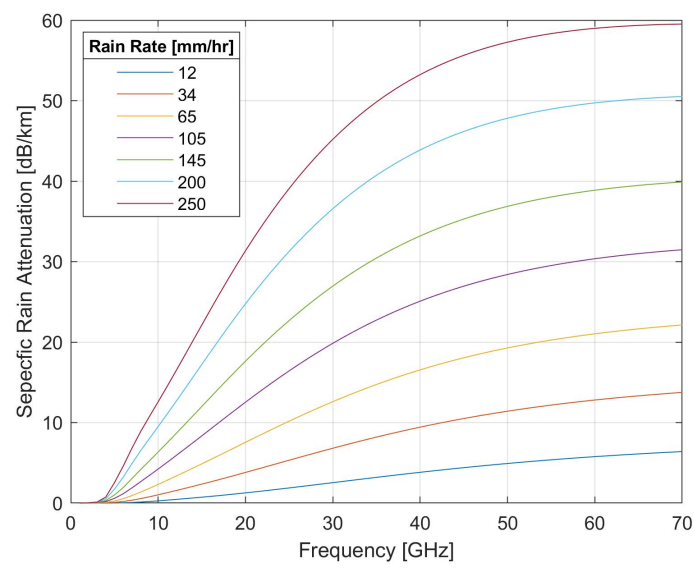

Figure 7. Rain attenuation versus different frequencies for different rain rate values registered during one year in Malaysia.

\subsection{Outdoor to Indoor Path Loss Model}

The outdoor-to-indoor scenario considers the deployment of UAV as an aerial base station in providing wireless coverage to outdoor and indoor users. The path loss model discussed in Section 2.1 is not appropriate for providing a coverage for indoor users, where this model assumes that all users are outdoor and located at 2D points. The outdoor-to-indoor path loss model that is certified by the International Telecommunication Union (ITU) [31] when considering the case of providing wireless coverage for indoor receivers only.

However, in the case of operating frequencies at higher bands, the wall penetration loss, $L_{t w}$, is derived and included in the outdoor-to-indoor path loss model. Moreover, in this study, the effect of rain attenuation to 5G spectrum in Malaysia is studied. Therefore, 
the rain attenuation, $L_{p}$ of Equation (11) is also included in the outdoor-to-outdoor path loss model as follows: the path loss for an outdoor-to-indoor model that considers wall penetration loss is given as:

$$
L_{I}=L_{F S P}+L_{t w}+L_{i n}+L_{p}
$$

where $L_{t w}$ is given by Equation (23). $L_{i n}=0.5 * d_{2 d}, d_{2 d}$ is the horizontal distance between the incidence on building wall and the indoor user. $L_{F S P}$ is given by:

$$
L_{F S P}=20 \log \left(\frac{4 \pi f_{c}}{c}\right)+20 \log \left(d_{3 d}\right)+P(\operatorname{LoS}) * \eta_{\text {LoS }}+(1-P(\operatorname{LoS})) * \eta_{N L o S}
$$

where $d_{3 d}$ is the 3D distance between the UAV and an indoor user.

\subsubsection{Wall Penetration Loss}

This section presents the derivation of wall penetration loss by analyzing the two mechanisms causing this additional loss which are transmission at the air dielectric interface, and the absorption that occurs when the wave traverses the wall. In this work, the derivation of wall penetration loss considers operating frequency at higher bands. In addition, the derivation of wall penetration loss assumes that a wall consists of two materials, namely concrete and glass.

The wall transmission loss will be analyzed assuming a single interface between air and the wall material where the transmission coefficient depends on the frequency and the real part of the dielectric constant of the wall material [32]. On the other hand, absorption coefficient is caused by the imaginary part of the dielectric constant [32].

The real part of the relative permittivity varies with frequency according to:

$$
\epsilon_{r 2}^{\prime}=a f^{b}
$$

where $f$ is the frequency in $\mathrm{GHz}$, and $a$ and $b$ are factors depending on the wall material as shown in $[29,32]$.

The imaginary part of the relative permittivity is related to the material conductivity $\sigma$ as follows:

$$
\epsilon_{r 2}^{\prime \prime}=\frac{\sigma}{2 \pi f \epsilon_{0}}
$$

where $\epsilon_{0}$ is the permittivity of free space and $\sigma$ varies with frequency according to:

$$
\sigma=c f^{d}
$$

where $f$ is the frequency in $\mathrm{GHz}$, and $c$ and $d$ are factors depending on the wall material as shown in [32].

The attenuation rate in $\mathrm{dB} / \mathrm{m}$ of the dielectric material is given as:

$$
A_{\text {dielectric }}=1636 \frac{\sigma}{\sqrt{\epsilon_{r 2}^{\prime}}}
$$

The attenuation due to the conductivity of wall material will be:

$$
A_{\sigma}=\frac{A_{\text {dielectric }} d}{\cos \theta} \text { in } \mathrm{dB}
$$

where $d$ is the wall thickness and $\theta$ is the angle of incidence on the wall. The total loss due to wall penetration (assuming circular polarization) is given as:

$$
L_{t w}=A_{\sigma}+\frac{-10 \log _{10} T_{s T M}-10 \log _{10} T_{S T E}}{2}
$$


where $T_{S T M}$ and $T_{S T E}$ are power transmission coefficient of the magnetic and electric fields, respectively [32].

Equation (19) and parameters value in Table 3 are used to plot Figure 8a,b. Figure 8a shows the penetration loss of a concrete wall of thickness $30 \mathrm{~cm}$ as a function of the angle of incidence at different frequencies, whereas Figure $8 \mathrm{~b}$ shows the penetration loss of glass material of thickness $2 \mathrm{~cm}$ as a function of the angle of incidence at different frequencies.

Table 3. Material properties [32].

\begin{tabular}{cccccc}
\hline Material Class & \multicolumn{2}{c}{ Real Part of Relative Permittivity } & \multicolumn{2}{c}{ Conductivity } & Frequency Range \\
\hline & $\mathbf{a}$ & $\mathbf{b}$ & $\mathbf{c}$ & $\mathbf{d}$ & $\mathbf{G H z}$ \\
\hline Concrete & 5.31 & 0 & 0.0326 & 0.8095 & $1-100$ \\
Plasterboard & 2.94 & 0 & 0.0116 & 0.7076 & $1-100$ \\
Wood & 1.99 & 0 & 0.0047 & 1.0718 & $0.001-100$ \\
Glass & 6.27 & 0 & 0.0043 & 1.1925 & $0.1-100$ \\
\hline
\end{tabular}

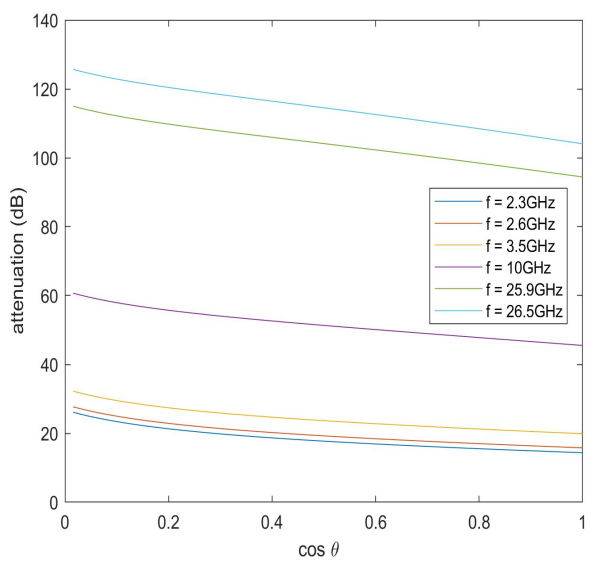

(a)

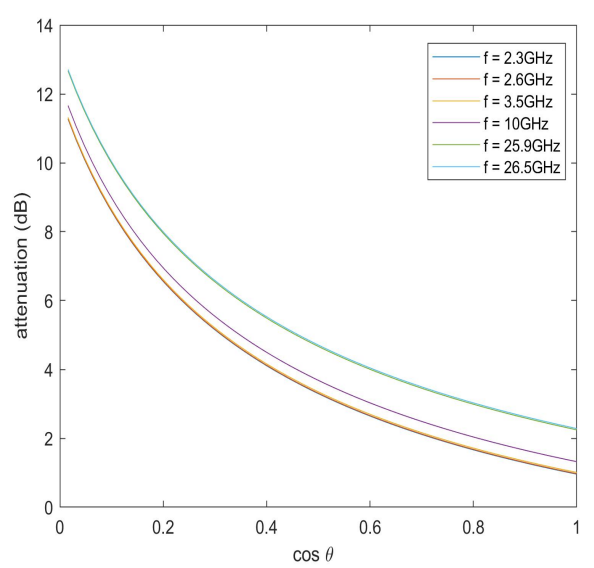

(b)

Figure 8. Penetration loss as a function of cosine of the angle of incidence. (a) for concrete wall; (b) for glass.

The penetration loss of a concrete wall and glass material, respectively, which can be represented in the form of:

$$
L_{t w}=b_{1}+b_{2}(1-\cos \theta)^{2}
$$

From Figure 8a,b, and in the frequency range from 1 to $60 \mathrm{GHz}$, the coefficients $\mathrm{b} 1$ and b2 are approximated as:

$$
\begin{aligned}
& b_{1}=b_{11}+b_{12} f \\
& b_{2}=b_{21}+b_{22} f
\end{aligned}
$$

The value of parameters $b_{11}, b_{12}, b_{21}$, and $b_{22}$ for concrete, and glass materials are derived from Figure $8 \mathrm{a}, \mathrm{b}$ and are listed in Table 4.

Table 4. Loss parameters for concrete and glass materials.

\begin{tabular}{ccccccc}
\hline \multirow{2}{*}{ Material Class } & \multirow{2}{*}{ Material Thickness } & \multicolumn{4}{c}{ Loss } & \multicolumn{2}{c}{ Frequency Range } \\
\cline { 3 - 6 } & & $\boldsymbol{b}_{\mathbf{1 1}}$ & $\boldsymbol{b}_{\mathbf{1 2}}$ & $\boldsymbol{b}_{\mathbf{2 1}}$ & $\boldsymbol{b}_{\mathbf{2 2}}$ & (GHz) \\
\hline Concrete & $30 \mathrm{~cm}$ & 16.0762 & 3.0993 & 10.9247 & 0.3783 & $1-60$ \\
Glass & $2 \mathrm{~cm}$ & 0.5821 & 0.1295 & 10.9247 & 0.3783 & $1-60$ \\
\hline
\end{tabular}


For a wall area divided in the ratio $p \%$ glass and $(1-p) \%$ concrete, wall penetration in $\mathrm{dB}$ can be approximated as $[25,33]$ :

$$
L_{t w}=-10 \log _{10}\left(p 10^{-\frac{L_{t w-g l a s s}}{10}}+(1-p) 10^{-\frac{L_{t w-\text { concrete }}}{10}}\right)
$$

Wall Penetration Loss Validation

Penetration loss of Equation (20) is validated by comparing it with the results presented by the authors in [34] at $60.5 \mathrm{GHz}$ for indoor-to-indoor and outdoor-to-indoor mobile scenarios. More specifically, in [34], the attenuation versus incidence angle attenuation for two different materials (single layer), namely, wood, and glass were plotted. The thickness of the single-layered wood and glass materials was set as $45 \mathrm{~mm}$ and $9.5 \mathrm{~mm}$, respectively. Figure 9 shows Equation (20) curves for wood and glass material at $60.5 \mathrm{GHz}$, which is in agreement with that of [34]. Thus, this validates the derivation of penetration loss term of Equation (20).

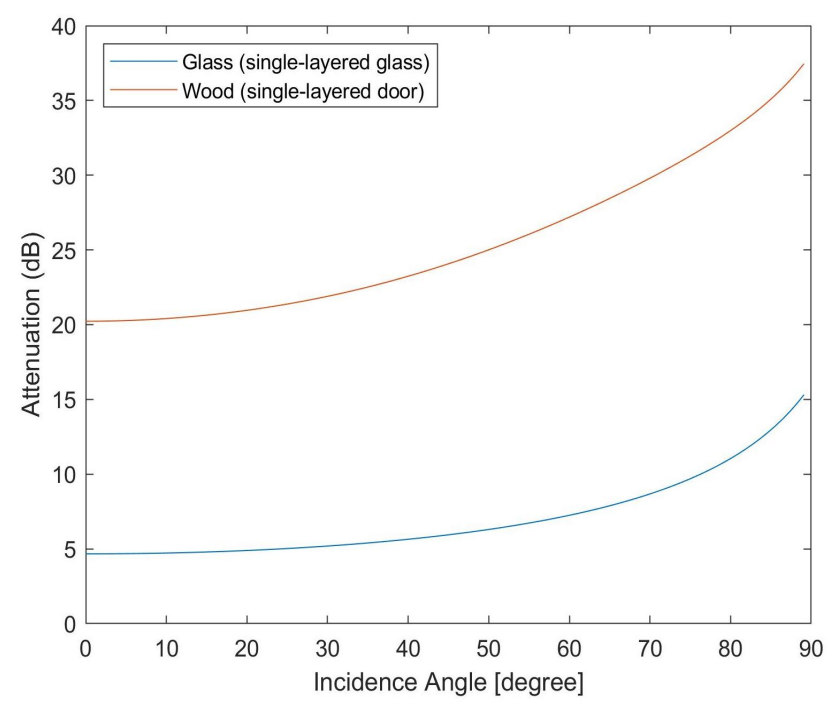

Figure 9. Attenuation in $\mathrm{dB}$ versus incidence angle at $60.5 \mathrm{GHz}$ using a penetration loss term of Equation (20) for glass and wood.

\subsection{Optimum 3D Placement of $U A V$}

The problem of finding the optimum UAV 3D placement is formulated with the objective to minimize the total path loss between UAV and users and is given as:

$$
\begin{gathered}
\text { minimize } \\
x_{\text {uav }}, y_{\text {uav }}, z_{\text {uav }}
\end{gathered}
$$

subject to

$$
\begin{aligned}
& x_{\text {min }} \leq x_{U A V} \leq x_{\text {max }}, \\
& z_{\text {min }} \leq y_{U A V} \leq y_{\text {max }}, \\
& z_{\text {min }} \leq z_{U A V} \leq z_{\text {max }}
\end{aligned}
$$

whereas $L_{\text {worst-case }}$ user is the maximum path loss of all the users.

The formulated problem is non-convex, and, due to its intractability, two algorithms are developed based on gradient descent (GD) and particle swarm optimization (PSO) algorithms.

Gradient descent is an optimization algorithm that is used when training a machine learning model. It is based on a convex function and tweaks its parameters iteratively to minimize a given function to its local minimum. For example, in the case of an upside parabola, for gradient descent to reach the local minimum and avoid bouncing, a learning rate is set to an appropriate value. 
Gradient descent starts at the initialized point, and it takes one step after another in the steepest downside direction until it reaches the point where the cost function is as small as possible. In this study, the input values are the initial location of UAV, the step size $\alpha_{s}$ which is the learning rate, gradient termination tolerance, minimum allowed perturbation, and the maximum allowed number of iterations. It begins to calculate the derivative of cost function from which then the learning rate will be updated.

The pseudo-code of the steps of this technique is given in Algorithm 1. During the while loop, the new location is calculated by multiplying the derivative of cost function with $\alpha_{s}$, which is the step size shown in step 7 of Algorithm 1. The while loop will be terminated if the derivative of the cost function is larger than or equal to tolerance, difference of previous location from new location larger than or equal to the allowed change (dxmin), or when it reaches the end of the maximum allowed number of iterations shown in step 8 of Algorithm 1.

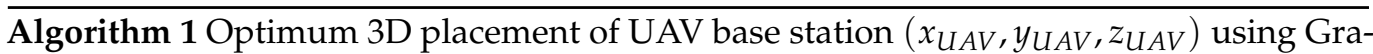
dient Descent algorithm

1: Input:

2: The 3D locations of the users and the cell dimensions

3: The step size $\alpha_{s}$, minimum allowed perturbation $\left\|\left(d x_{U A V}, d y_{U A V}, d z_{U A V}\right)\right\|_{\text {min }}$, and the gradient termination tolerance tol.

4: The maximum number of iterations max - iter

5: Initialize: $x_{U A V}, y_{U A V}, z_{U A V}$

6: For $n=1$ to max - iter

7: $x_{U A V, n+1}, y_{U A V, n+1}, z_{U A V, n+1} \leftarrow x_{U A V, n}-\alpha_{s} \frac{d C}{d x_{U A V}}, y_{U A V, n}-\alpha_{s} \frac{d C}{d y_{U A V}}, z_{U A V, n}-\alpha_{S} \frac{d C}{d z_{U A V}}$

8: IF $\left\|x_{U A V, n+1}-x_{U A V, n}, y_{U A V, n+1}-y_{U A V, n}, z_{U A V, n+1}-z_{U A V, n}\right\|$ $<\left\|\left(d x_{U A V}, d y_{U A V}, d z_{U A V}\right)\right\|_{\text {min }}$ and $\left\|\frac{d C}{d x_{U A V}}, \frac{d C}{d y_{U A V}}, \frac{d C}{d z_{U A V}}\right\|<$ tol

9: Return: $x_{U A V, o p t}, y_{U A V, o p t}, z_{U A V, o p t}=x_{U A V, n+1}, y_{U A V, n+1}, z_{U A V, n+1}$ End FOR

PSO is based on the paradigm of the swarm intelligence, and it is inspired by the social behavior of animals like flocks of birds, and schools of fish, which move together when searching for food [35]. Thus, in this work, the PSO algorithm is assumed to have a certain number of virtual UAVs which is randomly distributed in the area where the simulation is examined. These virtual UAVs constitute the members of the swarm which will move and communicate together towards the best solution with the aim to minimize the objective function.

Algorithm 2 presents the pseudo code of the PSO algorithm to find the optimized UAV 3D placement in providing wireless coverage.

Step 1 in Algorithm 2 presents the inputs of the algorithm, namely, N_pop defines the population of candidate solutions (virtual UAVs) of the algorithm, $W$ refers to the inertia weight, while $r_{1}$ and $r_{2}$ denote the two random numbers uniformly distributed random in a range between 0 and 1 , and $c_{1}$ and $c_{2}$ are the acceleration coefficients.

In the initialization step, the values of constriction factor, $\kappa$, cognitive parameter, $\phi_{1}$, and the social parameter, $\phi_{2}$ must be selected, where $\kappa=1$, and $\phi_{1}+\phi_{2}>4$ [35], which results in finding the efficient solution for the formulated problem. The PSO algorithm is initialized with a group of random solutions for all particles' positions and particles' velocities, as in steps 4 to 11 of Algorithm 2. Then, in every iteration, the local best location and the velocity for each particle are updated. In addition, the global best location is updated also, as in steps 12 to 22 of Algorithm 2. This is expected to move the virtual UAV swarm towards the best solution. 


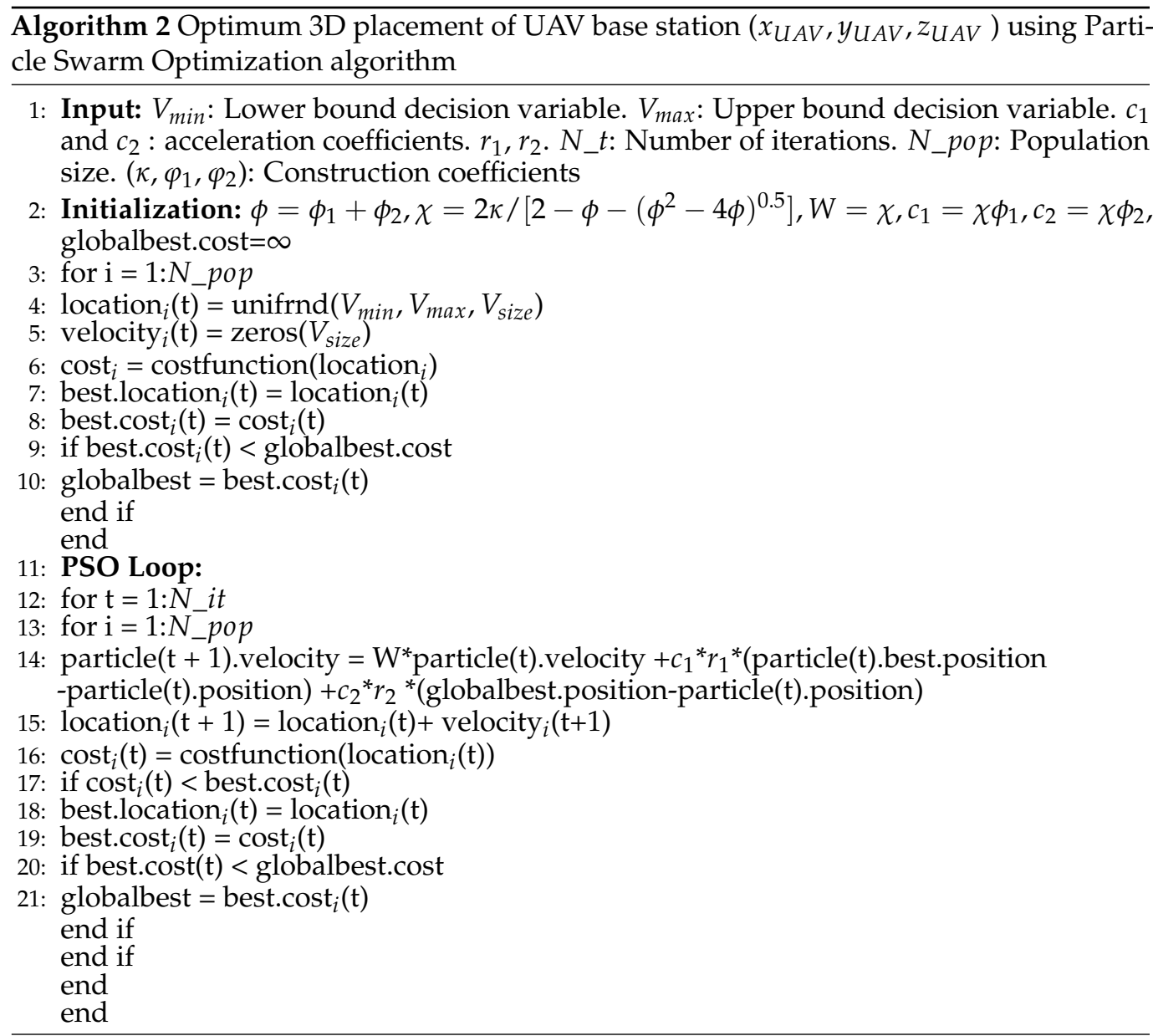

The optimum path loss is then used to calculate the transmit power using the Shannon theorem. Shannon theorem is given as:

$$
C=B \log _{2}\left(1+P_{r} / N_{p}\right)
$$

where $C$ is the bit rate available for a user, $B$ is the allocated bandwidth to each user, $N_{p}$ is the noise power superimposed on the signal received by the user's cellular phone, and $P_{r}$ is the received power by the user's cellular phone.

Focusing on the user with the worst condition, whose path loss is maximum, and with threshold value for bit rate, $C_{t h}$, then the user minimum received power is given as:

$$
P_{r-\min }=N_{p}\left[2^{\left(\frac{C_{t h}}{B}\right)}-1\right]
$$

Then, the minimum UAV transmit power is given as:

$$
P_{t-\min }=P_{r-\min }+L_{\max }
$$

where $L_{\text {max }}$ is the maximum loss encountered by the worst case user in the coverage area served by the UAV.

\section{Simulation Results}

This section presents the simulation results of the proposed GD and PSO algorithms to find the optimized UAV 3D placement in providing wireless coverage to users in two scenarios. The parameters used in the simulations are outlined in Tables 5-7. 
Table 5. General simulation parameters.

\begin{tabular}{cc}
\hline Parameters & Value \\
\hline No. of outdoor users & 75 \\
No. of floors & 20 \\
No. of users per floor & 30 \\
Height of floor & $4 \mathrm{~m}$ \\
Data Rate & $64 \mathrm{kbps}$ \\
Noise power & $-120 \mathrm{dBm}$ \\
Analog Bandwidth per user & $10 \mathrm{KHz}$ \\
\hline
\end{tabular}

Table 6. Simulation Parameters for PSO algorithm.

\begin{tabular}{cc}
\hline Parameters & Value \\
\hline$\left(V_{\min }, V_{\text {max }}, V_{\text {size }}\right)$ & $(0,100,3)$ \\
Population size $\left(N_{-} p o p\right)$ & 30 \\
Max. no. of iterations, $\left(N_{-} i t\right)$ & 50 \\
$\left(\kappa, \varphi_{1}, \varphi_{2}\right)$ & $(1,2.05,2.05)$ \\
Damping ratio & 1 \\
\hline
\end{tabular}

Table 7. Simulation Parameters for GD algorithm.

\begin{tabular}{cc}
\hline Parameters & Value \\
\hline Tolerance & $1 \times 10^{-6}$ \\
Max. no. of iterations & 500 \\
Minimum Perturbation & $5 \times 10^{-6}$ \\
Alpha $\left(\alpha_{s}\right)$ & 500 \\
\hline
\end{tabular}

\subsection{Outdoor-to-Outdoor Scenario}

This scenario considers a UAV to serve 75 outdoor users at the frequency of $28.1 \mathrm{GHz}$ and a rain rate $=250 \mathrm{~mm} / \mathrm{h}$. The two proposed algorithms GD and PSO algorithms are used to find the optimized UAV 3D placement in providing wireless coverage to 75 outdoor users. Figure 10a,b show the effect of rain rate on the UAV transmit power for different operating frequencies using the proposed GD algorithm, whilst the same analysis using the proposed PSO algorithm is shown in Figure 11a,b.

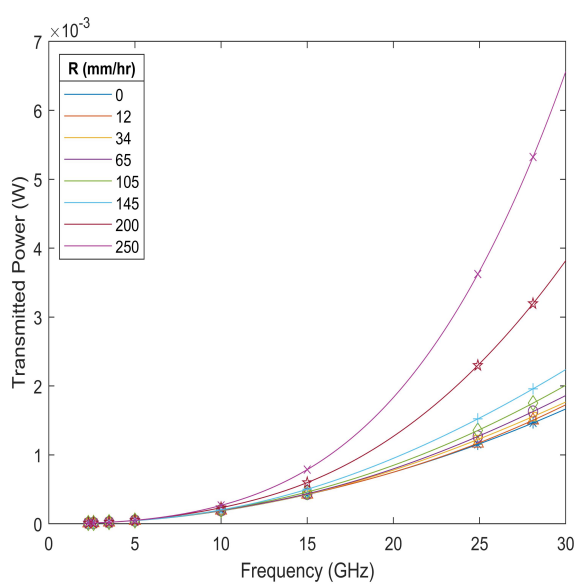

(a)

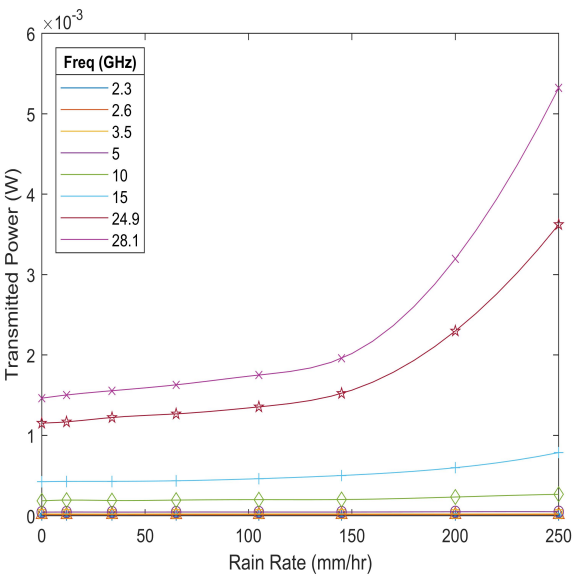

(b)

Figure 10. UAV transmit power using the proposed GD algorithm. (a) UAV transmit power versus operating frequency for different rain rate; (b) UAV transmit power versus rain rate for different operating frequencies. 


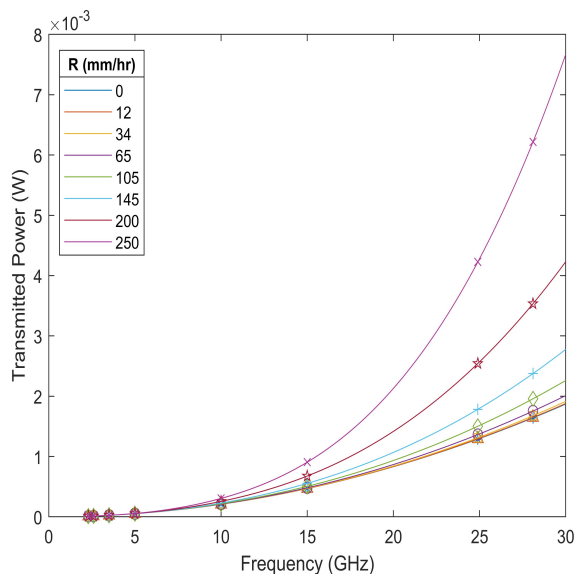

(a)

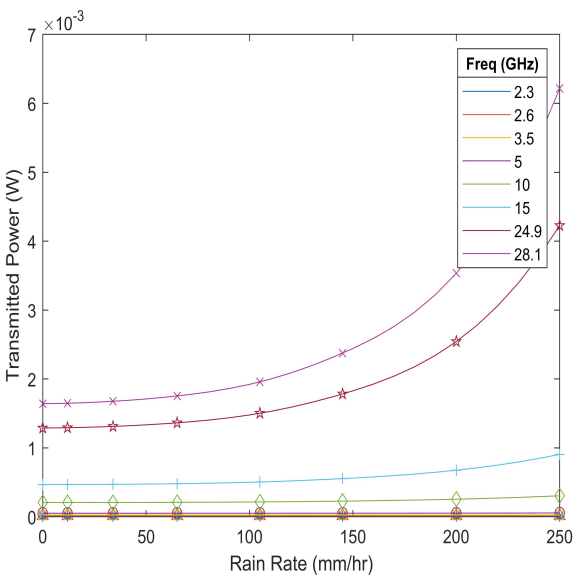

(b)

Figure 11. UAV transmit power using the proposed PSO algorithm (a) AV transmit power versus operating frequency for different rain rate; (b) UAV transmit power versus rain rate for different operating frequencies.

Figure 12 shows the distribution of outdoor users inside the coverage area, denoted by the blue dots and the red cross denotes the 2D location of UAV that is found using the proposed PSO algorithm. The proposed GD algorithm resulted in the same 2D location of UAV.

Table 8 presents UAV 3D placement using the proposed GD and PSO algorithms to serve the uniformly distributed outdoor users for rain rate values of 0 and $250 \mathrm{~mm} / \mathrm{h}$, at frequencies of $3.5 \mathrm{GHz}, 24.9 \mathrm{GHz}$, and $28.1 \mathrm{GHz}$. It can be observed that at $3.5 \mathrm{GHz}$ the presence of rain does not cause propagation loss. This is reflected in the UAV required transmit power of $25.4 \mu \mathrm{W}$ for both cases with and without the presence of rain at the rate of $250 \mathrm{~mm} / \mathrm{h}$. However, at $28.1 \mathrm{GHz}$, the presence of rain at the rate of $250 \mathrm{~mm} / \mathrm{h}$ resulted in an increase of UAV required transmit power by a factor of 4 compared to the case when there is no rain. Moreover, the optimal UAV 3D placement is identical using both the proposed GD and PSO algorithms for the case of outdoor-to-outdoor scenario. This observation is in line with the results shown in Figure 6 where rainfall contributed significant rain attenuation at frequencies of $24.9 \mathrm{GHz}$ and $28.1 \mathrm{GHz}$.

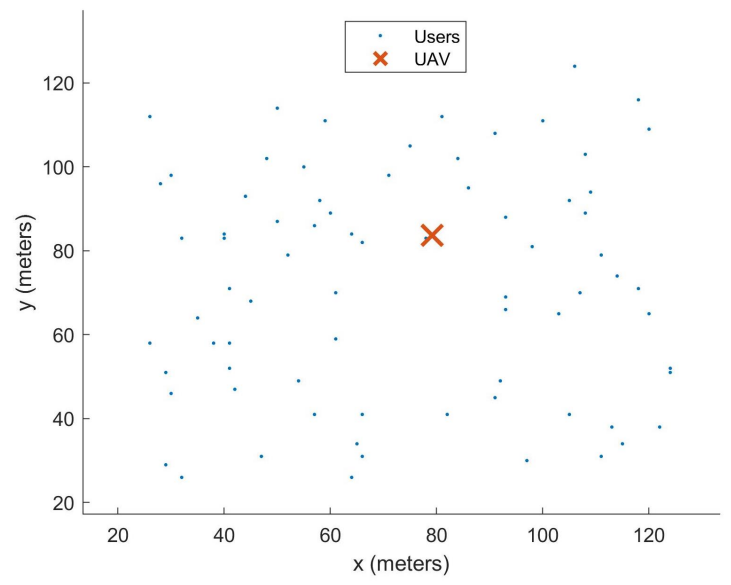

Figure 12. Uniform distribution of 75 outdoor users with the optimum UAV location for $\mathrm{f}=28.1 \mathrm{GHz}$ and $\mathrm{R}=250 \mathrm{~mm} / \mathrm{h}$ using the proposed PSO algorithm. 
Table 8. Simulation results for outdoor-to-outdoor scenarios.

\begin{tabular}{ccccc}
\hline $\begin{array}{c}\text { Rain Rate } \\
(\mathbf{m m} / \mathbf{h})\end{array}$ & $\begin{array}{c}\text { Freq } \\
(\mathbf{G H z})\end{array}$ & Algorithm & $\begin{array}{c}\text { Optimized 3D UAV Placement } \\
\left(x_{U A V}, y_{U A V}, z_{U A V}\right)\end{array}$ & $\begin{array}{c}\text { UAV Transmit Power } \\
\text { (watt) }\end{array}$ \\
\hline \multirow{4}{*}{3.5} & PSO & $(75.88,69.05,100)$ & $2.54 \times 10^{-5}$ \\
& & GD & $(75.91,68.99,94.92)$ & $2.37 \times 10^{-5}$ \\
0 & 24.9 & PSO & $(75.88,69.05,100)$ & 0.0013 \\
& & GD & $(75.91,68.96,92.04)$ & 0.0012 \\
& 28.1 & PSO & $(75.89,69.05,100)$ & 0.0016 \\
& GD & $(75.91,68.95,91.85)$ & 0.0015 \\
\hline \multirow{2}{*}{250} & PSO & $(75.88,69.05,100)$ & $2.56 \times 10^{-5}$ \\
& \multirow{2}{*}{2.5} & GD & $(75.90,68.97,93.43)$ & $2.33 \times 10^{-5}$ \\
& \multirow{2}{*}{28.9} & PSO & $(75.85,69.14,100)$ & 0.0042 \\
& & GD & $(75.87,69.07,92.77)$ & 0.0036 \\
& & PSO & $(75.85,69.15,100)$ & 0.0062 \\
\end{tabular}

\subsection{Outdoor-to-Indoor Scenario}

This scenario considers a UAV to serve 75 outdoor users and 2400 indoor users at the frequency of $28.1 \mathrm{GHz}$ and a rain rate $=250 \mathrm{~mm} / \mathrm{h}$. Figure 13 shows the 3D view of the distribution of outdoor and indoor users in the coverage area. The UAV transmit power for the worst-case user path loss using the data rate of $64 \mathrm{kbps}$ and noise power $-120 \mathrm{dBm}$ are plotted against frequency and rain rate as shown in Figures 14 and 15.

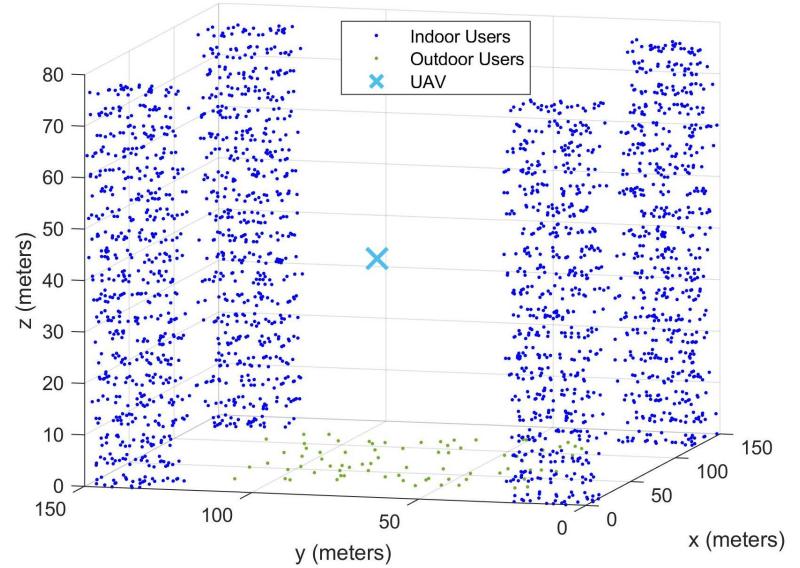

Figure 13. 3D view of 75 outdoor users and 2400 indoor users with the optimum UAV location for $\mathrm{f}=28.1 \mathrm{GHz}$ and $\mathrm{R}=250 \mathrm{~mm} / \mathrm{h}$.

Table 9 presents UAV 3D placement using the proposed GD and PSO algorithms to serve the uniformly distributed outdoor and indoor users simultaneously for rain rate values of 0 and $250 \mathrm{~mm} / \mathrm{h}$, at frequencies of $3.5 \mathrm{GHz}, 24.9 \mathrm{GHz}$, and $28.1 \mathrm{GHz}$. It can be observed that at $3.5 \mathrm{GHz}$ the presence of rain resulted in a small propagation loss. This is reflected in the small increment of UAV required to transmit power by a factor of 2 . However, at $28.1 \mathrm{GHz}$, the presence of rain at the rate of $250 \mathrm{~mm} / \mathrm{h}$ resulted in an increase of UAV required transmit power by a factor of 15 . In a similar observation at $24.9 \mathrm{GHz}$, the presence of rain at the rate of $250 \mathrm{~mm} / \mathrm{h}$ resulted in an increase of UAV required transmit power by a factor of 13. This observation is in line with the results shown in Figure 6 where rainfall contributes to significant rain attenuation at frequencies of $24.9 \mathrm{GHz}$ and $28.1 \mathrm{GHz}$.

It can also be observed that, for all cases, the optimal UAV 3D placement is not identical when using different proposed GD and PSO algorithms. More specifically, the UAV placement is located in the middle of the coverage area when using the proposed GD algorithm. However, the optimal UAV 3D placement using the proposed PSO algorithm resulted in less UAV transmit power compared to that when using the proposed GD 
algorithm. The discrepancies of the UAV transmit power can be explained by studying the mesh plot of the path loss equation as illustrated in Figure 16.

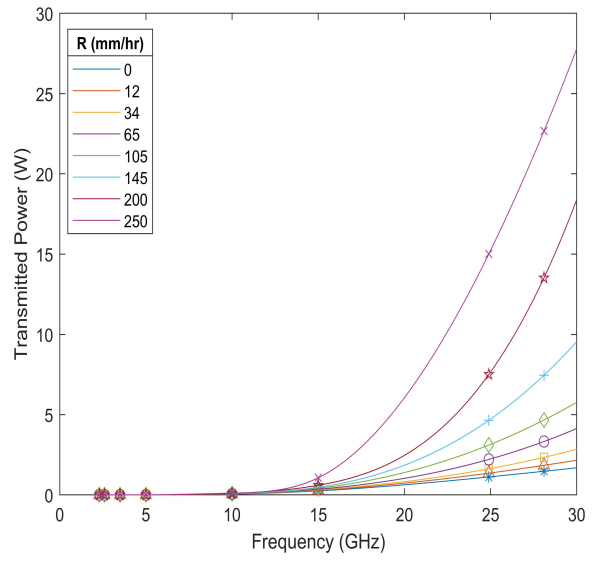

(a)

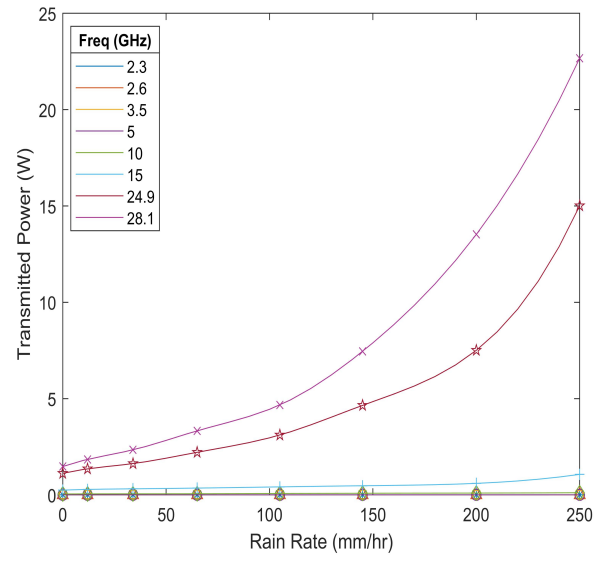

(b)

Figure 14. UAV transmit power using the proposed PSO algorithm. (a) UAV transmit power versus operating frequency for different rain rate; (b) UAV transmit power versus rain rate for different operating frequencies.

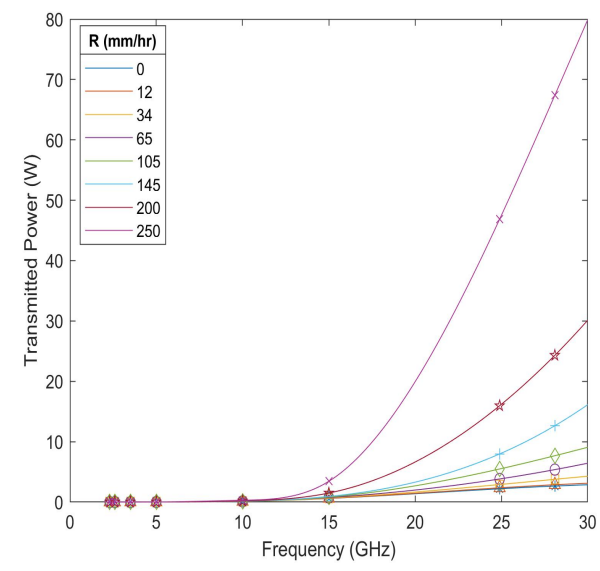

(a)

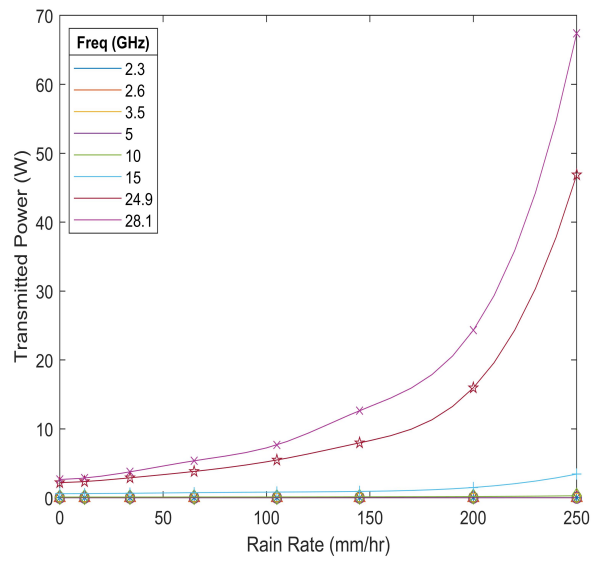

(b)

Figure 15. UAV transmit power using the proposed GD algorithm. (a) UAV transmit power versus operating frequency for different rain rate; (b) UAV transmit power versus rain rate for different operating frequencies.

Table 9. Simulation results for outdoor-to-indoor scenario.

\begin{tabular}{ccccc}
\hline $\begin{array}{c}\text { Rain Rate } \\
(\mathbf{m m} / \mathbf{h})\end{array}$ & $\begin{array}{c}\text { Freq } \\
(\mathbf{G H z})\end{array}$ & Algorithm & $\begin{array}{c}\text { Optimized 3D UAV Placement } \\
\left(x_{U A V}, y_{U A V}, z_{U A V}\right)\end{array}$ & $\begin{array}{c}\text { UAV Transmit Power } \\
\text { (Watt) }\end{array}$ \\
\hline \multirow{2}{*}{3.5} & PSO & $(94.88,77.37,40.05)$ & 0.0014 \\
& & GD & $(80.72,76.89,47.35)$ & 0.0055 \\
0 & 24.9 & PSO & $(89.93,73.70,38.37)$ & 1.1207 \\
& & GD & $(24.38,75.46,38.78)$ & 2.2038 \\
& 28.1 & PSO & $(79.09,78.50,38.60)$ & 1.4741 \\
& & GD & $(24.33,75.79,39.05)$ & 2.6601 \\
\hline \multirow{2}{*}{3.5} & PSO & $(94.63,77.19,40.10)$ & 0.0027 \\
& \multirow{2}{*}{250} & GD & $(75.59,76.25,47.07)$ & 0.0073 \\
& \multirow{2}{*}{28.9} & PSO & $(79.46,83.33,37.99)$ & 15.013 \\
& & GD & $(75.10,77.96,43.42)$ & 46.871 \\
& \multirow{2}{*}{28.1} & PSO & $(79.06,86.77,38.56)$ & 22.672 \\
& & GD & $(72.97,78.82,43.31)$ & 67.398 \\
\hline
\end{tabular}


Figure 16 shows the mesh plot of the path loss equation at $28.1 \mathrm{GHz}$ and rain rate of $250 \mathrm{~mm} / \mathrm{h}$ for UAV coverage area with dimensions of $x=25 \mathrm{~m}$ and $y=100 \mathrm{~m}$ ( $x$ - and $y$-axis, respectively). It can be observed that there is a number of local minima within the UAV coverage area in the mesh plot; thus, the gradient descent algorithm in the outdoor-to-outdoor and outdoor-to-indoor scenario was unable to converge to the global minimum as its approach to find the solution is affected by the initial values which resulted in sub-optimal solution.

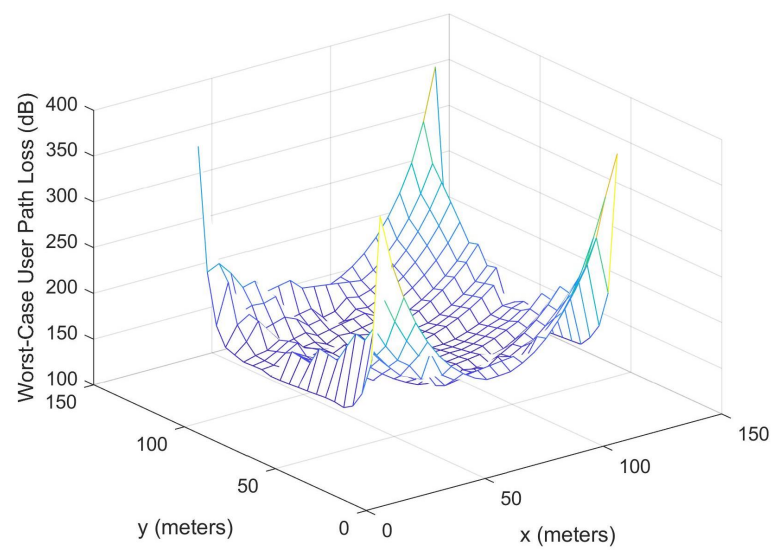

Figure 16. Mesh plot for the path loss equation for the coverage area of $x=25 \mathrm{~m}$ and $y=100 \mathrm{~m}$ at operating frequencies of $28.1 \mathrm{GHz}$ and a rain rate of $250 \mathrm{~mm} / \mathrm{h}$.

\section{Conclusions}

In this study, the path loss models for outdoor-to-outdoor and outdoor-to-indoor scenarios when UAV is deployed as aerial base station are developed by considering user's antenna height, rain attenuation, and wall penetration loss. The problem of finding optimal UAV 3D placement is formulated with the objective to minimize the total path loss between the UAV and all users. The formulated problem is solved by developing two algorithms that invoke PSO and GD algorithms to find optimal UAV 3D placement for two scenarios, namely, outdoor-to-outdoor and outdoor-to-indoor scenarios. It is observed that, for the outdoor-to-outdoor scenario, both algorithms resulted in similar UAV 3D placement unlike for the outdoor-to-indoor scenario. It is also observed that, in both scenarios, the proposed algorithm that invokes PSO requires less iterations to converge to the minimum transmit power compared to that of the algorithm that invokes GD. Moreover, it is also observed that the rain attenuation increases the total path loss for high operating frequencies, namely at $24.9 \mathrm{GHz}$ and $28.1 \mathrm{GHz}$. Hence, this resulted in an increase in UAV required transmit power. At $28.1 \mathrm{GHz}$, the presence of rain at the rate of $250 \mathrm{~mm} / \mathrm{h}$ resulted in an increase of UAV required transmit power by a factor of 4 and 15 for outdoor-to-outdoor and outdoorto-indoor scenarios, respectively. In our future work, the directivity of the antenna factor will be considered in the path loss model. Furthermore, real-time measurements will be conducted and compared with the simulation results.

Author Contributions: Conceptualisation, A.M.S. and N.S.O.; methodology, A.M.S. and N.S.O.; software, A.M.S.; validation, A.M.S. and N.S.O.; formal analysis, A.M.S. and N.S.O.; writing—original draft preparation, A.M.S. and N.S.O.; writing-review and editing, A.M.S., N.S.O.; supervision, N.S.O.; funding acquisition, N.S.O. All authors have read and agreed to the published version of the manuscript.

Funding: This research was funded by the Universiti Tenaga Nasional BOLD Research Grant 2020 (Grant No.: RJO10517844/102).

Conflicts of Interest: The authors declare no conflict of interest. 


\section{References}

1. Azmani, S.; Juliana, N.; Idrose, A.; Amin, N.; Saudi, A. Challenges of communication system during emergency disaster response in Malaysia: A review. J. Fundam. Appl. Sci. 2017, 9, 890-904. [CrossRef]

2. Sawalmeh, A.H.; Othman, N.S.; Shakhatreh, H.; Khreishah, A. Wireless Coverage for Mobile Users in Dynamic Environments Using UAV. IEEE Access 2019, 7, 126376-126390. [CrossRef]

3. Shakhatreh, H.; Sawalmeh, A.H.; Al-Fuqaha, A.; Dou, Z.; Almaita, E.; Khalil, I.; Othman, N.S.; Khreishah, A.; Guizani, M. Unmanned Aerial Vehicles (UAVs): A Survey on Civil Applications and Key Research Challenges. IEEE Access 2019, 7, 48572-48634. [CrossRef]

4. Bupe, P.; Haddad, R.; Rios-Gutierrez, F. Relief and emergency communication network based on an autonomous decentralized UAV clustering network. In Proceedings of the SoutheastCon 2015, Fort Lauderdale, FL, USA, 9-12 April 2015; pp. 1-8. [CrossRef]

5. Bor-Yaliniz, R.I.; El-Keyi, A.; Yanikomeroglu, H. Efficient 3D placement of an aerial base station in next generation cellular networks. In Proceedings of the 2016 IEEE International Conference on Communications, ICC 2016, Kuala Lumpur, Malaysia, 23-27 May 2016. [CrossRef]

6. Uragun, B. Energy efficiency for unmanned aerial vehicles. In Proceedings of the 10th International Conference on Machine Learning and Applications, ICMLA 2011, Honolulu, HI, USA, 18-21 December 2011. [CrossRef]

7. Sawalmeh, A.; Othman, N.S.; Shakhatreh, H.; Khreishah, A. Providing wireless coverage in massively crowded events using UAVs. In Proceedings of the 2017 IEEE 13th Malaysia International Conference on Communications, MICC 2017, Johor Bahru, Malaysia, 28-30 November 2017; pp. 158-163. [CrossRef]

8. Shakhatreh, H.; Khreishah, A.; Alsarhan, A.; Khalil, I.; Sawalmeh, A.; Othman, N.S. Efficient 3D placement of a UAV using particle swarm optimization. In Proceedings of the 2017 8th International Conference on Information and Communication Systems, ICICS 2017, Irbid, Jordan, 4-6 April 2017. [CrossRef]

9. Shakhatreh, H.; Khreishah, A.; Khalil, I. Indoor Mobile Coverage Problem Using UAVs. IEEE Syst. J. 2018, 12, 3837-3848. [CrossRef]

10. Mozaffari, M.; Saad, W.; Bennis, M.; Debbah, M. Drone small cells in the clouds: design, deployment and performance analysis. In Proceedings of the 2015 IEEE Global Communications Conference, GLOBECOM 2015, San Diego, CA, USA, 6-10 December 2015. [CrossRef]

11. Mozaffari, M.; Saad, W.; Bennis, M.; Debbah, M. Efficient Deployment of Multiple Unmanned Aerial Vehicles for Optimal Wireless Coverage. IEEE Commun. Lett. 2016, 20, 1647-1650. [CrossRef]

12. Mozaffari, M.; Saad, W.; XBennis, M.; Debbah, M. Optimal transport theory for power-efficient deployment of unmanned aerial vehicles. In Proceedings of the 2016 IEEE International Conference on Communications, ICC 2016, Kuala Lumpur, Malaysia, 23-27 May 2016. [CrossRef]

13. Zeng, H.; Zhu, X.; Jiang, Y.; Wei, Z.; Sun, S. Toward UL-DL Rate Balancing: Joint Resource Allocation and Hybrid-Mode Multiple Access for UAV-BS Assisted Communication Systems. arXiv 2021, arXiv:2111.08233.

14. Shakhatreh, H.; Khreishah, A.; Ji, B. Providing wireless coverage to high-rise buildings using UAVs. In Proceedings of the 2017 IEEE International Conference on Communications (ICC), Paris, France, 21-25 May 2017.

15. Sawalmeh, A.; Othman, N.S.; Shakhatreh, H. Efficient deployment of multi-UAVs in massively crowded events. Sensors 2018, 18, 3640. [CrossRef] [PubMed]

16. Zeng, Y.; Zhang, R. Energy-efficient UAV communication with trajectory optimization. IEEE Trans. Wirel. Commun. 2017, 16, 3747-3760. [CrossRef]

17. Lam, H.Y.; Luini, L.; Din, J.; Alhilali, M.J.; Jong, S.L.; Cuervo, F. Impact of rain attenuation on 5G millimeter wave communication systems in equatorial Malaysia investigated through disdrometer data. In Proceedings of the 2017 11th European Conference on Antennas and Propagation, EUCAP 2017, Paris, France, 19-24 March 2017. [CrossRef]

18. Kestwal, M.C.; Joshi, S.; Garia, L.S. Prediction of rain attenuation and impact of rain in wave propagation at microwave frequency for tropical region (Uttarakhand, India). Int. J. Microw. Sci. Technol. 2014, 2014, 958498. [CrossRef]

19. Rappaport, T.S.; MacCartney, G.R.; Samimi, M.K.; Sun, S. Wideband millimeter-wave propagation measurements and channel models for future wireless communication system design. IEEE Trans. Commun. 2015, 63, 3029-3056. [CrossRef]

20. Shayea, I.; Abd Rahman, T.; Hadri Azmi, M.; Islam, M.R. Real Measurement Study for Rain Rate and Rain Attenuation Conducted over 26 GHz Microwave 5G Link System in Malaysia. IEEE Access 2018, 6, 19044-19064. [CrossRef]

21. Shayea, I.; Nissirat, L.A.; Nisirat, M.A.; Alsamawi, A.; Abd. Rahman, T.; Hadri Azmi, M.; Abo-Zeed, M.; Trrad, I. Rain attenuation and worst month statistics verification and modeling for $5 \mathrm{G}$ radio link system at $26 \mathrm{GHz}$ in Malaysia. Trans. Emerg. Telecommun. Technol. 2019, 30, e3697. [CrossRef]

22. ITU. Recommendation ITU-R P.838-3: Specific Attenuation Model for Rain; Recommendation ITU-R P.838; ITU: Geneva, Switzerland, 2005; pp. 1-8.

23. Khawaja, W.; Guvenc, I.; Matolak, D.W.; Fiebig, U.C.; Schneckenburger, N. A Survey of Air-to-Ground Propagation Channel Modeling for Unmanned Aerial Vehicles. IEEE Commun. Surv. Tutor. 2019, 21, 2361-2391. [CrossRef]

24. Al-Hourani, A.; Kandeepan, S.; Lardner, S. Optimal LAP altitude for maximum coverage. IEEE Wirel. Commun. Lett. 2014, 3, 569-572. [CrossRef]

25. Matolak, D.W.; Sun, R. Air-Ground Channel Characterization for Unmanned Aircraft Systems-Part III: The Suburban and Near-Urban Environments. IEEE Trans. Veh. Technol. 2017, 66, 6607-6618. [CrossRef] 
26. Matolak, D.W. Unmanned aerial vehicles: Communications challenges and future aerial networking. In Proceedings of the 2015 International Conference on Computing, Networking and Communications (ICNC), Garden Grove, CA, USA, 16-19 February 2015; pp. 567-572. [CrossRef]

27. Al-Hourani, A.; Kandeepan, S.; Jamalipour, A. Modeling air-to-ground path loss for low altitude platforms in urban environments. In Proceedings of the 2014 IEEE Global Communications Conference, GLOBECOM 2014, Austin, TX, USA, 8-12 December 2014; pp. 2898-2904. [CrossRef]

28. Valcarce, A.; Rasheed, T.M.; Gomez, K.M.; Sithamparanathan, K.; Reynaud, L.; Hermenier, R.; Munari, A.; Mohori, M.; Smolnikar, M.; Bucaille, I. Airborne Base Stations for Emergency and Temporary Events. In Proceedings of the 5th International Conference on Personal Satellite Services, PSATS 2013, Toulouse, France, 27-28 June 2013; Springer: Cham, Switzerland, 2013.

29. ITU-R. Propagation Data and Prediction Methods Required for the Design of Terrestrial Broadband Radio Access Systems in a Frequency Range from 3 to $60 \mathrm{GHz}$; Rec. ITU-R P.1410-5; ITU: Geneva, Switzerland, 2012; Volume 5.

30. Seybold, J.S. Introduction to RF Propagation; John Wiley \& Sons: New York, NY, USA, 2005; pp. 1-330. [CrossRef]

31. Series, M. Guidelines for Evaluation of Radio Interface Technologies for IMT-Advanced; Report ITU; ITU: Geneva, Switzerland, 2009; Volume 638, pp. 1-72.

32. ITU-R-Radiocommunication Sector of ITU. Recommendation ITU-R P.2040-1: Effects of Building Materials and Structures on Radiowave Propagation above About 100 MHz; Recommendation ITU-R P.2040; ITU: Geneva, Switzerland, 2015 ; Volume 1.

33. ITU. Guidelines for Evaluation of Radio Interface Technologies for IMT-2020; Report ITU-R M.2412; ITU: Geneva, Switzerland, 2017.

34. Jun, S.Y.; Caudill, D.; Chuang, J.; Papazian, P.B.; Bodi, A.; Gentile, C.; Senic, J.; Golmie, N. Penetration Loss at 60 GHz for Indoor-to-Indoor and Outdoor-to-Indoor Mobile Scenarios. In Proceedings of the 14th European Conference on Antennas and Propagation, EuCAP 2020, Copenhagen, Denmark, 15-20 March 2020; pp. 6-10. [CrossRef]

35. Kennedy, J.; Eberhart, R. Particle swarm optimization. In Proceedings of the IEEE International Conference on Neural Networks, Perth, WA, Australia, 27 November-1 December 1995. [CrossRef] 